\title{
Optimization of dew point sensitive structure based on a quartz resonant sensor
}

\author{
Yi Yang ${ }^{1,}$,, Xiaofeng Meng ${ }^{1, b}$, Qiang Wang ${ }^{3, c}$ \\ ${ }^{1}$ Department of Measurement Control and Information Technology, School of Instrumentation \\ Science and Opto-electronics Engineering, Beihang University, Beijing 100191, China \\ ayiyangbuaa@163.com, ${ }^{\mathrm{b}}$ mengxf@buaa.edu.cn
}

Keywords: Sensitive structure; Quartz crystal; Optimization; Simulation.

Abstract: A new design of dew point sensitive structure based on a quartz resonant humidity sensor is proposed. The resonant frequency of the quartz crystal is considered as a humidity sensitive parameter in Humidity measurement method. Combined with Peltier module, active condensation occurs in the surface of quartz crystal, the variation of its resonant frequency identifies the time of dew point. Optimization of dew point sensitive structure based on a quartz resonant sensor is mainly discussed, the mechanical properties and temperature characteristics of the combination mode consist of the quartz crystal, semiconductor refrigerator and the temperature sensor was analyzed, which was in different medium and with different viscous material. For the optimization of the sensitive structure, the design of the sensitive structure, the software simulation of the sensitive structure and a test experiment system of the sensitive structure mode was considered as the main work.

\section{Introduction}

Humidity is the amount of water vapor in the air. As a significant parameter of atmosphere, humidity has influenced on human life. And achieving a humidity sensitive structure with high accuracy, low cost and a wide range of applications has always been the domestic and international difficult problem [1,2].

Recently for the sake of a humidity sensitive device with high sensitivity, rapid response, fast recovery and small hysteresis, many efforts have been paid. And some achievements have been made $[3,4,5]$. This topic mainly explored the feasibility of sensitive structure designed and its measuring results on the basis of quartz crystal humidity measurement method, which laid a foundation for further realization of practical application.

The sensitive structure of dew point sensor based on quartz resonator is mainly composed of three parts: the quartz crystal sensor, semiconductor cooler and temperature sensor. The quartz crystal is the center part of this device. Combined with semiconductor cooler, active condensation occurs in the surface of quartz crystal to change the mass on it, the variation of its resonant frequency identifies the time of condensation while the temperature sensor gets the dew point temperature simultaneously [7]. The semiconductor cooler controls the temperature of quartz crystal, and the temperature sensor was dedicated to real-time monitor the temperature variation of the quartz crystal. In order to get great thermal effect and highly accuracy of measurement, the quartz crystal should keep close connection with semiconductor cooler and temperature sensor. On the other hand, it also should be given further assurance to keep its stable resonance performance.

\section{Sensitive Structure Design}

In summary, combined with the physics response of humidity sensitive element, the optimization of sensitive structure covers 3 points:

$\square 1$. The combination mode between semiconductor cooler and quartz crystal. The unreasonable combination mode may lead to great effect on its resonant performance or even cease its resonance. A logical combination mode is indispensable which brings about great heat transfer and little influence on the resonant performance of quartz crystal. 
2. Accurate measurement of the temperature is an important indicator in system design. The temperature sensor should report the surface temperature of quartz crystal as much as possible when dew point time comes. In general, temperature sensor should keep close contact with measured object in order to get accuracy measurements. But in this device, settled the temperature sensor directly into the quartz crystal may cause great effects on its resonant frequency. While settled the temperature sensor into the surface of semiconductor cooler, the temperature of semiconductor cooler replaced the temperature of quartz crystal. A temperature difference existed between actual quantity and measurements that brought about an error in result. A logical sensor location is indispensable, which gives rise to little influence on resonant performance of quartz crystal and real-time monitor to the temperature of quartz crystal accurately.

3. For the high refrigerating efficiency, heat sink radiation is needed. Choosing an appropriate cooling device is important.

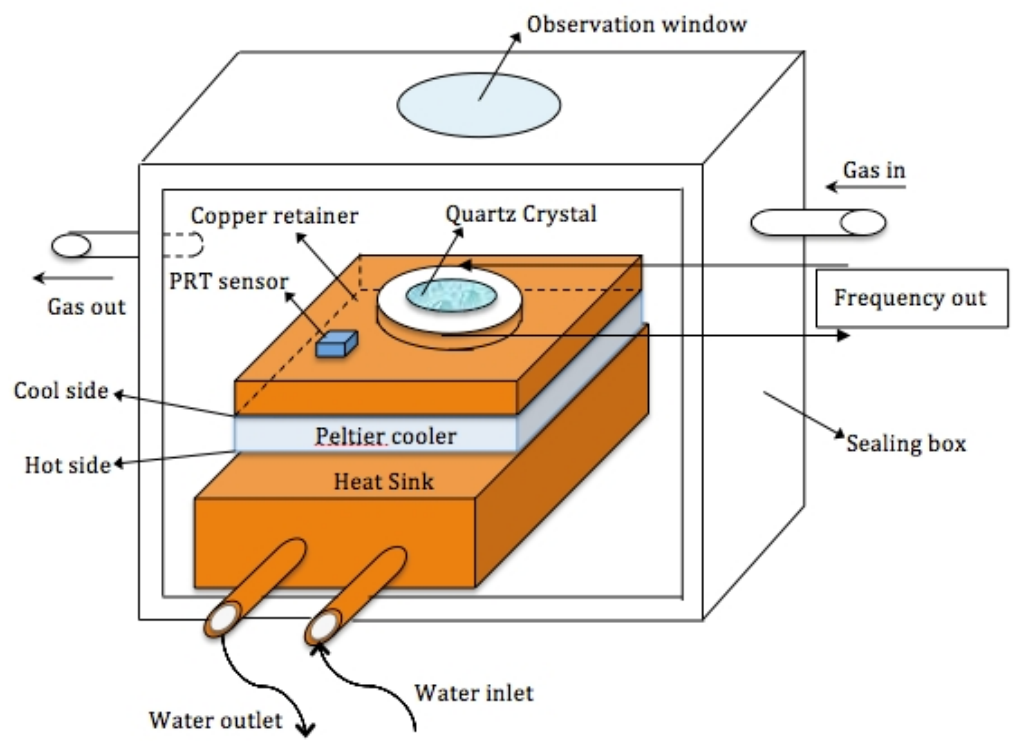

Figure1. Schematic diagram of sensitive structure

This sensitive device is made up of an 8.4-millimetre-diameter AT-cut quartz crystal with two symmetrical silver electrodes operating at a resonance frequency of $6 \mathrm{MHz}$. A copper retainer was placed in a fixed location to live in the middle between the quartz crystal and the semiconductor cooler (TEC-12706). That was used to form a heat-conducting connection between the quartz crystal and Peltier element, the semiconductor cooler. This quartz does not require any absorbent material, and it is directly stuck on the copper retainer. The contact between the quartz and the copper plate is made up of a circular ring of viscous material that has high heat conducting in order to improve thermal transfer. The Peltier module allows the cooling of the quartz crystal indirectly through the copper retainer until the dew point reached. When it occurs, water drops appear on the quartz, then the apparent mass of quartz crystal is modified and its resonant frequency decreases.

Figure1 shows a schematic diagram of the sensitive structure. In order to keep the resonant quality of quartz crystal, the temperature sensor that measures dew point temperature should avoid fixing directly on the surface of quartz crystal. And from the point of manufacturing process, it's also difficult to fix the temperature sensor directly on the surface of quartz crystal. The temperature sensor was settled on the surface of the copper plate that closes to quartz crystal with heat-conducting glue. The copper retainer, which has high-efficiency and homogeneous heat conducting, provided great thermal contact between quartz crystal and the temperature sensor. That is to say, A PT100, the temperature sensor, was placed on the copper retainer in order to follow the temperature of the copper, which replaced the temperature of quartz crystal.

A water-cooling system was used to the heat dissipation of the hot side of the semiconductor cooler. This water-cooling heat sink radiation is composed of an outlet pipe, an inlet pipe and some water channels inside. Water from water receiver was pumped by water pump, and then it flowed into water 
tank via inlet pipe, and flowed out through the outlet pipe, at last, back to water receiver. Cycling constantly like this, the advantage of this water-cooling system was totally taken. And more heat was taken away.

\section{Software Simulation}

Resonant Quality of Quartz Crystal. An 8.4-millimetre-diameter, 0.277-millimetre-thickness AT-cut quartz crystal was chosen as target in this simulation. And we used ANSYS as our finite element analysis (FEA) software. By the energy trapping theory, the synchronous wave could spread in electrode district, and dying out in non-electrode district. The great mass of vibration energy was limited in the electrode district of quartz crystal. That is to say, the location of viscous material on quartz crystal should be settled in the non-electrode district. Glues with different material characteristic was settled on the upside surface of quartz crystal, after model analysis by ANSYS, the most appropriate viscous material was confirmed. Considered the generalized, this simulation model chose a ring form model as the bonding layer in non-electrode district.

The Poisson ratio and the Young modulus are important parameters of material characteristic. The chart below shows the impacts on the resonant quality of quartz crystal by the Poisson ratio and the Young modulus of viscous material.

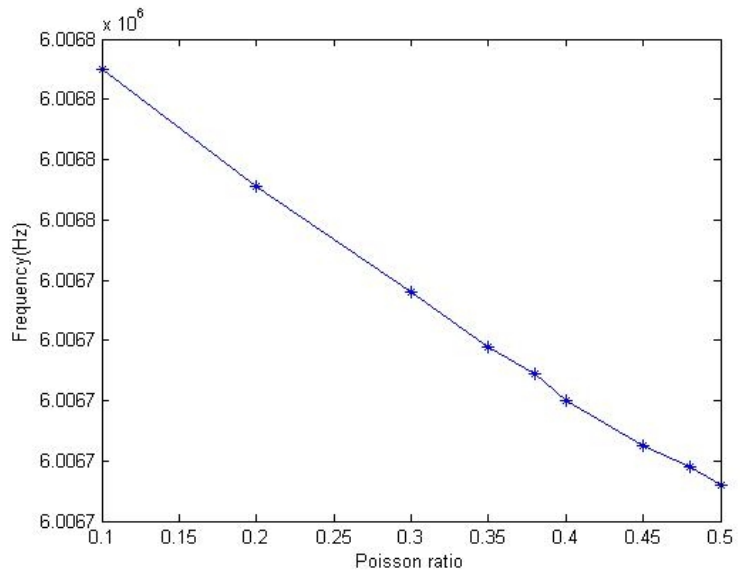

Figure2a. Poisson ratio-resonant frequency curve

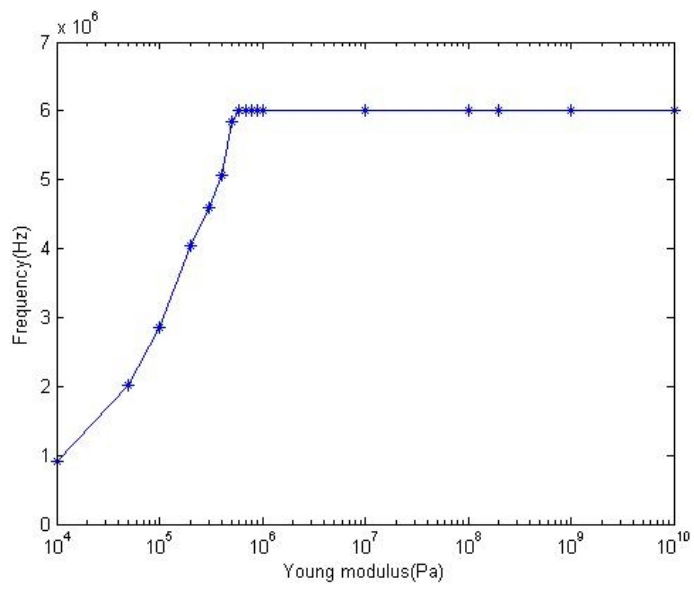

Figure2b. Young modulus-resonant frequency curve

From the Poisson ratio-resonant frequency curve (Fig.2a), the Poisson ratio just has a little effect on the resonant quality of quartz crystal. And it's in reverse proportion to the resonant quality in general. The Young modulus-resonant frequency curve (Fig.2b) tells that, when the Young modulus is less than $1 \mathrm{MPa}$, the Young modulus has a great influence on the resonant quality of quartz crystal, and it is proportional to the resonant frequency as in whole. While the Young modulus is more than $1 \mathrm{MPa}$, the quartz crystal could mainly keep its resonant frequency. To sum up, this structure could use conductive silver glue as viscous material.

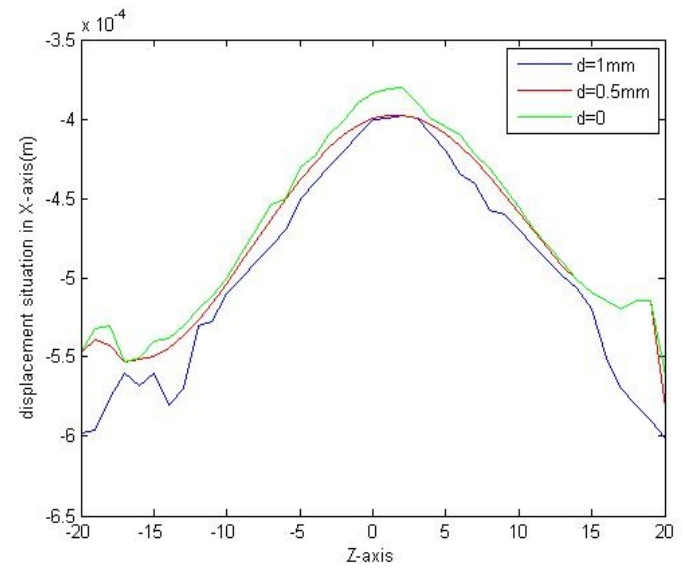

Figure3.Impacts of location on resonant quality 
The location (the distance to the edge of quartz crystal) of bonding layer in non-electrode district causes great impact on quartz crystal. The chart in Fig3 shows the displacement situation in $\mathrm{x}$-axis of every pot in $\mathrm{z}$-axis $(\mathrm{x}=0, \mathrm{y}=0.277)$ radial direction. This curve tells that, the more the bonding layer near the electrode district, the greater influence it has on quartz crystal. In another word, the distance of the bonding layer from the electrode district is negatively correlated to the impact on quartz crystal.

Fig4,5 tells the effects on the quartz crystal caused by the area and thickness of bonding layer. The bigger the area or thickness is, the greater influence on resonant quality it has. But in case of delamination, it`s appropriate to choose an area of $12.4 \mathrm{~mm}$ and a thickness of $0.06 \mathrm{~mm}$.

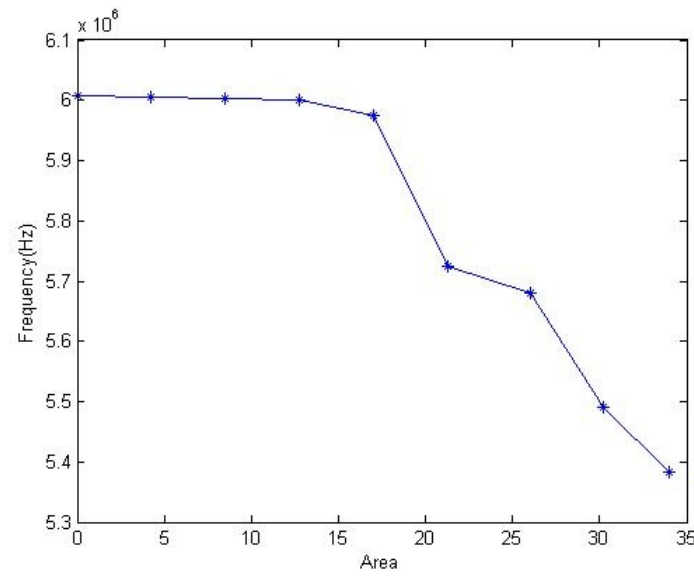

Figure4.Impacts of area on resonant quality

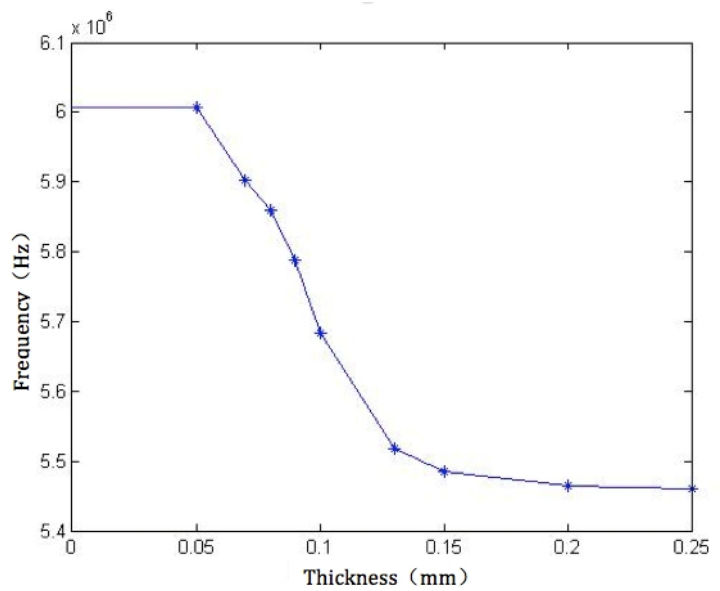

Figure5.Impacts of thickness on resonant quality

Thermal analysis of sensitive device. The Steady Thermal Analysis of humidity sensitive element mainly used for analyzing the temperature difference between quartz crystal surface and copper retainer, and the temperature difference among the pots in the moisture surface of quartz crystal when entered a steady state. This sensitive structure corresponds a symmetrical characteristic in space and load. Fig6 shows the thermal analysis model:

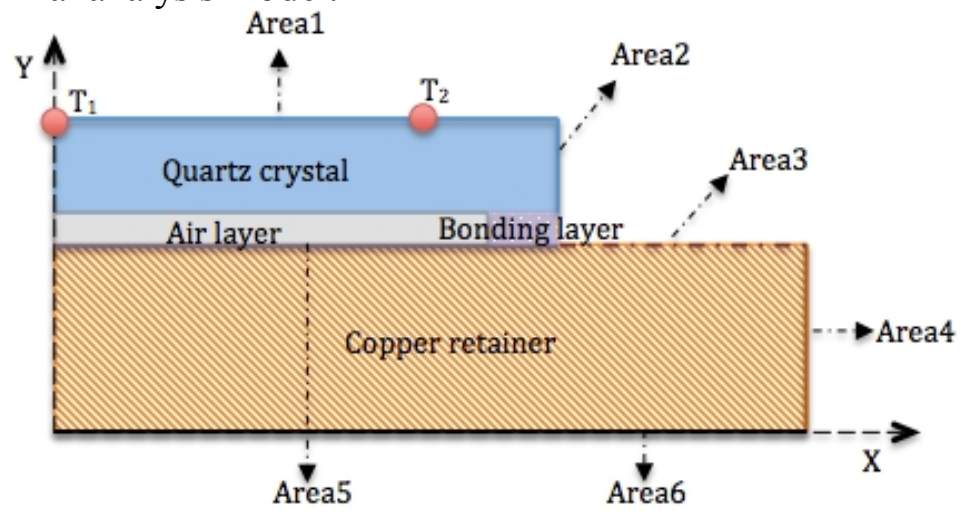

Figure6. Schematic diagram of thermal model

By applying the convective heat-transfer coefficient with air on Area1、2、3、4, and fixed temperature load $\mathrm{T}_{\text {load }}$ on Area6. The chart above (Fig7) shows the temperature of the pots on Area1. The biggest temperature difference in electrode district is:

$$
\left|T_{1}-T_{2}\right|=0.03234^{\circ} \mathrm{C}
$$

The surface temperature of quartz crystal could be replaced by the center temperature $T_{1}$ with this good uniformity. Fig8 shows the temperature of pots on copper retainer.

The Transient Analysis of humidity sensitive element mainly indicated the shift of time-dependent temperature difference between actual value and measurements under different temperature load $\mathrm{T}_{\text {load}}$. Be seen from the chart (Fig8), the temperature of the quartz crystal reached an equilibrium value with the temperature of the copper retainer within a period of about one minute through the copper contact mounting. Controlling only the temperature of the copper retainer instead of the quartz crystal was sufficient for our purpose. 


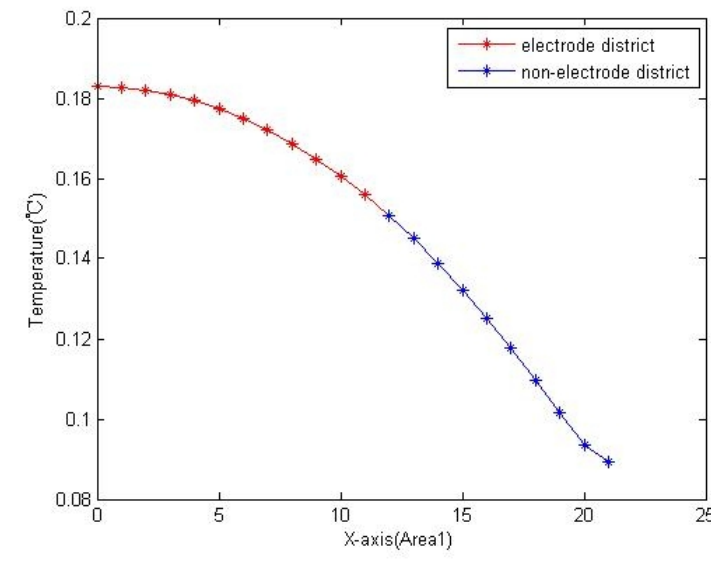

Figure7.Distribution of temperature on Area1

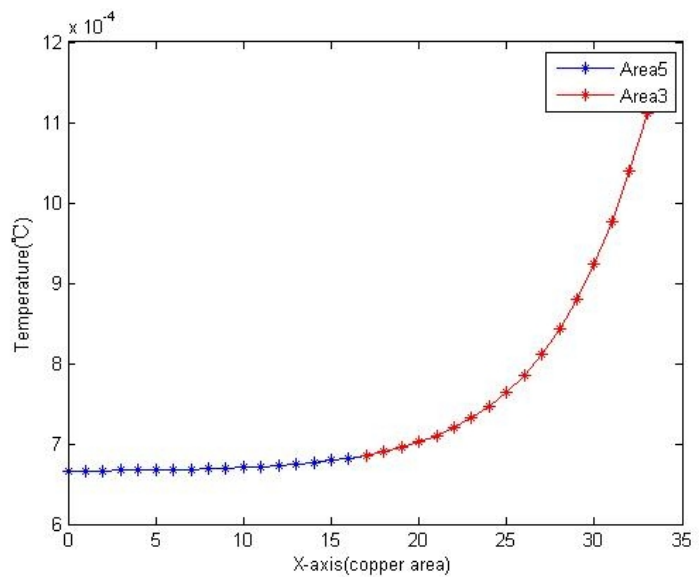

Figure8. Distribution of temperature on copper retainer

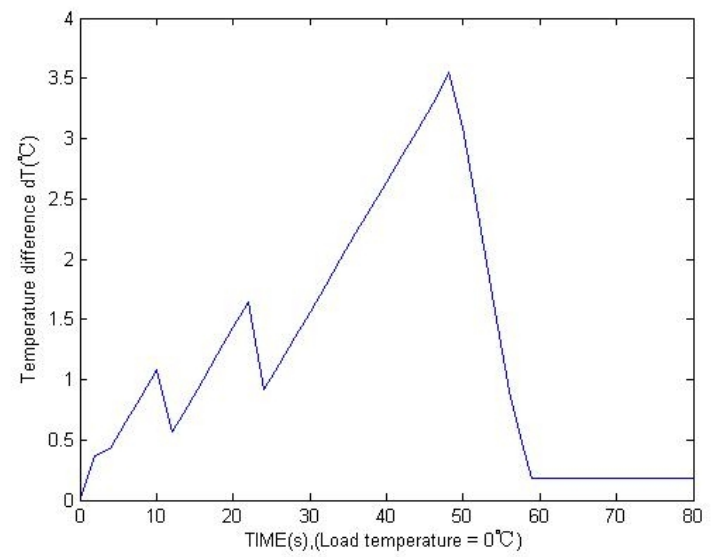

Figure9.Shift of time-dependent temperature difference between actual value and measurements

\section{Experimental Setup}

Diagram of experimental system is showed in Figure 9. In order to achieve the testing principle above, the hardware system used spectrum analyzer as frequency acquisition system that produced automatic scanning sine signal, and 4015 module for the temperature measurement of platinum resistor (RTD). Moreover, the whole sensitive structure was placed in a confined chamber, and a constant temperature was used to keep an environment with stable temperature. An aspirator pump system respectively pumped in moist air with same properties for this sensitive sensor and MICHELL S4000 photoelectric hygrometer, which used for humidity reference quantity.

\section{Results and discussion}

4 type of connection mode between quartz crystal and copper retainer was set: hanging in the air, conductive silver glue, conductive silica, and conductive rubber. The experimental procedure is: Respectively measured the resonant frequency of these 4 types; Added unit mass of water on the surface of quartz crystal and then measured its resonant frequency variation of these a types respectively; Kept adding until it ceased.

The time-dependent resonant frequency shift in 4 different connection modes is given in Fig 10 . Be seen from the chart, the connection mode between quartz crystal and copper retainer with thermal-conducting silver glue is more sensitive to the dew point.

In Figure 11, the temperature-time curve indicates that, the temperature of quartz crystal or copper retainer reached a stable value in less than one minute, and the temperature difference is less than $0.2^{\circ} \mathrm{C}$.

This experimental result was consistent with the simulation result. 


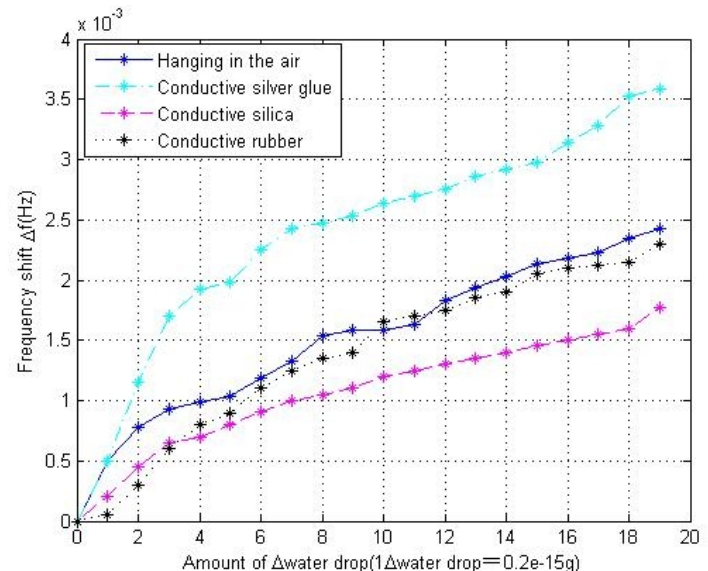

Figure10.Frequency shift with water drops in 4 type of connection mode

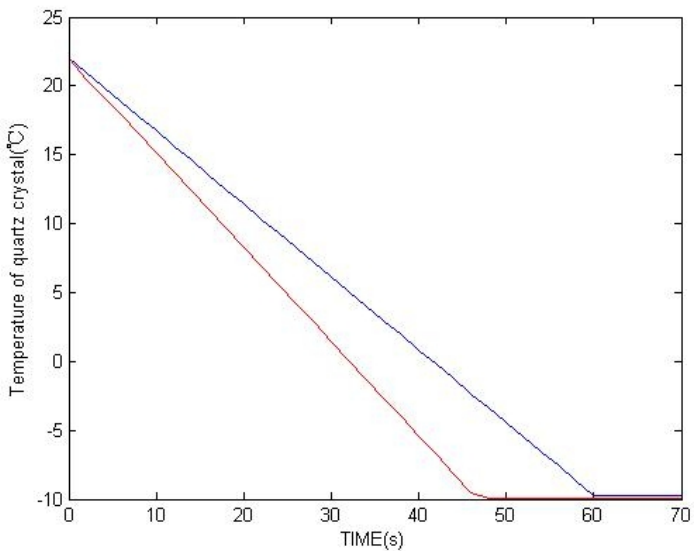

Figure1 1.Time-dependent temperature between actual value and measurements $\left(\mathrm{T}_{\text {load }}=-10^{\circ} \mathrm{C}\right)$

\section{Conclusion}

Compared with other similar devices, a new dew point sensitive structure based on a quartz resonant humidity sensor that has fast response time, high accuracy and high sensitivity is proposed. This sensitive device used a copper retainer to form a great heat-conducting connection between the quartz crystal and Peltier element. The quartz was directly pasted to the copper retainer with thermal-conducting silver glue. A PT100 was settled on the copper retainer. That is to say, the temperature of copper retainer replaced the temperature of quartz crystal. Therefore, the response time of this sensitive device achieved a period of less than one minute, and the temperature difference was less than $0.2^{\circ} \mathrm{C}$.

\section{Acknowledgement}

This research is funded by the National Natural Science Foundation of China (NSFC) under grant No. 61473021.

\section{References}

[1] Jing Nie,Xiaofeng Meng.Dew point and relative humidity measurement using a quartz resonant sensor [J].Microsystem Technologies.2013.DOI: 10.1007/s00542-013-1914-9.

[2] I. Dyer: Measurement of humidity. Anaesthesia \& Intensive Care Medicine, vol. 13, pp. 121-123, 2012.

[3] Kwon S Y, Kim J C. Accurate dew-point measurement over a wide temperature range using a quartz crystal microbalance dew-point sensor [J]. Meas. Sci. Technol, 2008, 19:115206.

[4] Ok J J, Young H K.Dew point measurement for organic vapor mixture using a quartz crystal sensor [J].Sensors and Actuators B,2006,113:335-340.

[5] Pascal-Delannoy F, Sorli B, Boyer A. Quartz Crystal Microbalance (QCM) used as humidity sensor [J]. Sensors and Actuators A, 2000,84:285-291.

[6] Jing Nie,Xiaofeng Meng,Rui Zheng,Shuo Wang.Fast resonant sensors for hight humidity range 60-95\%RH[J].Sensors \& Actuators: B. Chemica.2013 , 185:211-217. 